\title{
Topological Floquet edge states in periodically curved waveguides
}

\author{
Bo Zhu, ${ }^{1,2}$ Honghua Zhong, ${ }^{1,3}$ Yongguan Ke, ${ }^{1,2}$ Xizhou Qin, ${ }^{1}$ Andrey A. Sukhorukov, ${ }^{4}$ \\ Yuri S. Kivshar, ${ }^{4}$ and Chaohong Lee ${ }^{1,2,5, *}$ \\ ${ }^{1}$ Laboratory of Quantum Engineering and Quantum Metrology, School of Physics and Astronomy, \\ Sun Yat-Sen University (Zhuhai Campus), Zhuhai 519082, China \\ ${ }^{2}$ State Key Laboratory of Optoelectronic Materials and Technologies, Sun Yat-Sen University (Guangzhou Campus), \\ Guangzhou 510275, China \\ ${ }^{3}$ Institute of Mathematics and Physics, Central South University of Forestry and Technology, Changsha 410004, China \\ ${ }^{4}$ Nonlinear Physics Centre, Research School of Physics and Engineering, The Australian National University, Canberra ACT 2601, Australia \\ ${ }^{5}$ Synergetic Innovation Center for Quantum Effects and Applications, Hunan Normal University, Changsha 410081, China
}

(Received 11 April 2018; published 31 July 2018)

\begin{abstract}
We study the Floquet edge states in arrays of periodically curved optical waveguides described by the modulated Su-Schrieffer-Heeger model. Beyond the bulk-edge correspondence, our study explores the interplay between band topology and periodic modulations. By analyzing the quasienergy spectra and Zak phase, we reveal that, although topological and nontopological edge states can exist for the same parameters, they cannot appear in the same spectral gap. In the high-frequency limit, we find analytically all boundaries between the different phases and study the coexistence of topological and nontopological edge states. In contrast to unmodulated systems, the edge states appear due to either band topology or modulation-induced defects. This means that periodic modulations may not only tune the parametric regions with nontrivial topology, but may also support novel edge states.
\end{abstract}

DOI: 10.1103/PhysRevA.98.013855

\section{INTRODUCTION}

Recently, topological photonics has emerged as a new approach to manipulate properties of light under continuous deformations [1]. Electromagnetic topological states have been found in both microwave [2-4] and optical [5-7] regimes. Similar to topological insulators for electrons, photonic topological insulators have also been created [1-16]. Beyond conventional topological phenomena in linear Hermitian systems, topological gap solitons have been found in nonlinear optical systems [17], and it was shown that topological states can survive in non-Hermitian systems [18]. Moreover, periodic modulations can bring several novel topological properties usually absent in their nonmodulated analogs [9,19-26].

Bulk-edge correspondence $[27,28]$ is a well-established principle for two-dimensional (2D) topological systems. It establishes the exact correspondence between bulk states subjected to periodic boundary conditions (PBCs) and edge states in the systems with open boundary conditions (OBCs). Up to now, topological edge states have been found in several 2D photonic systems $[10,13,29,30]$. However, for one-dimensional (1D) lattice models, edge states have been shown to appear in periodically modulated but nontopological lattices [31,32]. This suggests that edge states can be induced by either topology or periodic modulations. Here, we wonder whether topological and nontopological edge states may coexist and, if they may coexist, how to distinguish between topological and nontopological edge states.

\footnotetext{
*Corresponding author: lichaoh2@mail.sysu.edu.cn; chleecn @ gmail.com
}

In this work, we study the Floquet edge states (FESs) in arrays of periodically curved optical waveguides described by a periodically modulated Su-Schrieffer-Heeger (SSH) model [33]. We analyze the interplay between band topology and periodic modulations, and describe the coexistence of both topological and nontopological edge states supported by the same parameters. Our results show that, for a specific gap, the Zak phase $Z_{G_{m}}$ is either zero or $\pi$, so that the topological edge states appear only in the gap of $Z_{G_{m}}=\pi$. Through controlling both modulation frequency and amplitude, we may drive the system from nontopological to topological regime, and vice versa. We demonstrate analytically that periodic modulations induce a virtual defect at the boundary-the key mechanism for the formation of nontopological edge states.

The paper is organized as follows. In Sec. II, we introduce our physical model and derive its coupled-mode equations. In Sec. III, we calculate the quasienergy spectra under OBC. In Sec. IV, by employing the multiscale perturbation analysis, we give the effective coupled-mode equations and demonstrate that the periodic modulations can induce virtual defects at boundaries. The FESs include defect-free surface states and Shockley-like surface states, which are induced by virtual defects and the alternating strong and weak couplings between waveguides, respectively. In Sec. V, we analytically obtain the asymptotic phase boundary and numerically give the phase diagram of appearing FESs, respectively. We explore the topological nature of all FESs via the calculation of the bulk topological invariant Zak phase. We find that Shockley-like surface states are topological FESs and defect-free surface states are nontopological FESs. A brief summary is given in Sec. VI. 

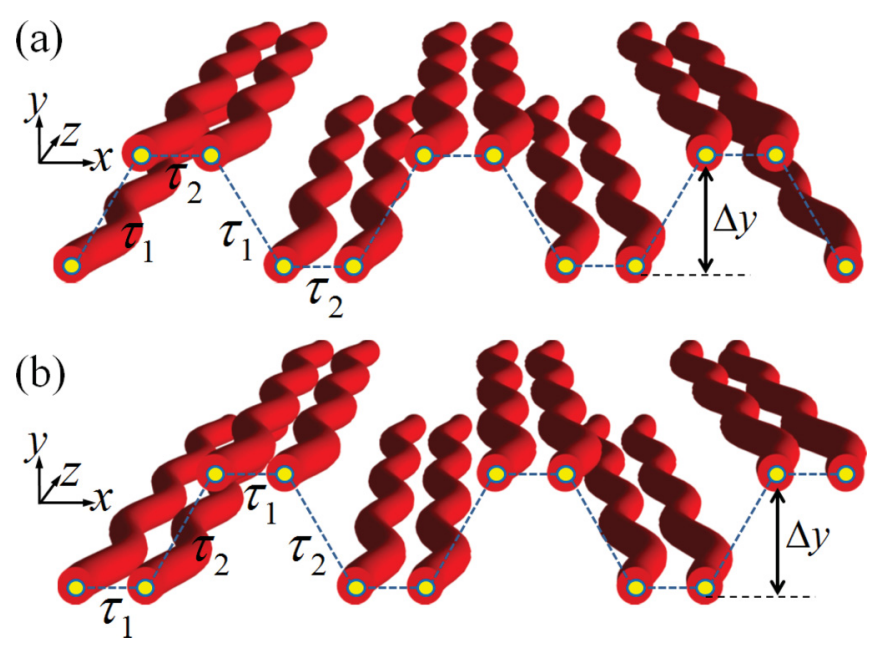

FIG. 1. Schematic diagram of waveguide arrays curved along the propagation direction of light ( $z$ axis). The center-to-center spacing along the $x$ axis is fixed as $\Delta x$, and the one along the $y$ axis is either zero or $\Delta y$ intermittently. The coupling strength is either $\tau_{1}$ or $\tau_{2}$ intermittently. (a) $\tau_{1} / \tau_{2}<1$ with $\tau_{2}=\tau$ and (b) $\tau_{1} / \tau_{2}>1$ with $\tau_{1}=\tau$.

\section{MODEL}

We consider an array of coupled optical waveguides, where the waveguides are periodically curved along the longitudinal propagation direction; see Fig. 1. The light field $\psi(x, y, z)$ obeys the paraxial wave equation

$$
-i \frac{\partial \psi}{\partial z}=\frac{\lambda^{\prime}}{4 \pi n^{\prime}}\left(\frac{\partial^{2}}{\partial x^{2}}+\frac{\partial^{2}}{\partial y^{2}}\right) \psi+\frac{2 \pi}{\lambda^{\prime}} v(x, y, z) \psi,
$$

where $\lambda^{\prime}$ is the optical wavelength in vacuum, $n^{\prime}$ is the medium refractive index, and $v(x, y, z)$ describes the refractive index at $(x, y, z)$. The waveguide centers $x_{n}(z)=x_{n}(z+T)$ are periodically curved along the longitudinal direction with the curving period $T$ much larger than the interwaveguide distance $\Delta x$. Here we set $x_{n}(z)=n \Delta x+A[\cos (\omega z)-1]$ with the modulation amplitude $A$ and the modulation frequency $\omega$.

By implementing the coordinate transformation $[\hat{z}=z, \hat{y}=$ $\left.y, \hat{x}(z)=x-x_{0}(z)\right]$, we have $\partial_{x}=\partial_{\hat{x}}, \partial_{y}=\partial_{\hat{y}}$, and $\partial_{z}=\partial_{\hat{z}}-$ $\dot{x}_{0} \partial_{\hat{x}}$. Therefore, the field $\psi(\hat{x}, \hat{y}, \hat{z})$ obeys

$$
-i \frac{\partial \psi}{\partial \hat{z}}=-i \dot{x}_{0} \frac{\partial \psi}{\partial \hat{x}}+\frac{2 \pi}{\lambda^{\prime}} \nu \psi+\frac{\lambda^{\prime}}{4 \pi n^{\prime}}\left(\frac{\partial^{2}}{\partial \hat{x}^{2}}+\frac{\partial^{2}}{\partial \hat{y}^{2}}\right) \psi
$$

By applying the gauge transformation

$$
\psi=\phi \exp \left\{i \frac{\pi n^{\prime}}{\lambda^{\prime}}\left(2 \dot{x}_{0}(\hat{z}) \hat{x}(\hat{z})-\int_{0}^{\hat{z}} \hat{x}_{0}^{2}(\xi) d \xi\right)\right\},
$$

the paraxial wave equation (1) can be written as

$$
-i \frac{\partial \phi}{\partial \hat{z}}=\frac{\lambda^{\prime}}{4 \pi n^{\prime}}\left(\frac{\partial^{2}}{\partial \hat{x}^{2}}+\frac{\partial^{2}}{\partial \hat{y}^{2}}\right) \phi+\frac{2 \pi}{\lambda^{\prime}} \nu \phi-\frac{2 \pi n^{\prime}}{\lambda^{\prime}} \ddot{x}_{0} \hat{x} \phi .
$$

Expanding the field into a superposition of the single-mode fields in individual waveguides

$$
\phi(\hat{x}, \hat{y}, \hat{z})=\sum_{n} \varphi_{n}(\hat{z}) a_{n}(\hat{x}, \hat{y})
$$

we obtain the coupled-mode equations

$$
-i \frac{d \varphi_{n}}{d z}=\tau_{n} \varphi_{n+1}+\tau_{n-1} \varphi_{n-1}+D_{n} \varphi_{n}-\eta \ddot{x}_{0} n \varphi_{n},
$$

where $\eta=2 \pi n^{\prime} / \lambda^{\prime}$ as a normalized optical frequency, and

$$
\begin{aligned}
\tau_{n} & =\frac{2 \pi}{\lambda^{\prime}} \iint a_{n}^{*}(\hat{x}, \hat{y}) v(\hat{x}, \hat{y}, \hat{z}) a_{n+1}(\hat{x}, \hat{y}) d \hat{x} d \hat{y}, \\
D_{n} & =\frac{2 \pi}{\lambda^{\prime}} \iint a_{n}^{*}(\hat{x}, \hat{y}) v(\hat{x}, \hat{y}, \hat{z}) a_{n}(\hat{x}, \hat{y}) d \hat{x} d \hat{y} .
\end{aligned}
$$

By performing a transformation

$$
\varphi_{n}=\exp \left[i \eta A \omega \hat{x}_{n} \sin (\omega z)+i D_{n} z\right] u_{n},
$$

we derive the coupled-mode equations as

$$
\begin{aligned}
-i \frac{d u_{n}}{d z}= & \tau_{n} \exp \left[i \eta A \omega\left(\hat{x}_{n+1}-\hat{x}_{n}\right) \sin (\omega z)\right] u_{n+1} \\
& +\tau_{n-1} \exp \left[-i \eta A \omega\left(\hat{x}_{n}-\hat{x}_{n-1}\right) \sin (\omega z)\right] u_{n-1}
\end{aligned}
$$

Here $\eta=2 \pi n^{\prime} / \lambda^{\prime} ; u_{n}$ denotes the complex field amplitude for the $n$th waveguide with $n$ being the waveguide index. As the center-to-center waveguide spacing along the $x$ axis is constant (i.e., $\hat{x}_{n+1}-\hat{x}_{n}=\hat{x}_{n}-\hat{x}_{n-1}=\Delta \hat{x}=1$ ) and one along the $y$ axis is either zero or $\Delta y$ intermittently, the hopping strengths can be written as $\tau_{n}=\frac{1}{2}\left\{\left[1-(-1)^{n}\right] \tau_{1}+\left[1+(-1)^{n}\right] \tau_{2}\right\}$ and the maximum hopping strength $\tau=\max \left\{\tau_{1}, \tau_{2}\right\}$ is fixed. By adjusting the distance $\Delta \hat{y}$, one may tune the values of $\tau_{n}$.

Without loss of generality, we set $\eta=1$ and $\tau=1$. Therefore, the system can be described by the periodically modulated SSH-like Hamiltonian

$$
H(z)=\sum_{n=1}^{2 N}\left\{\tau_{n} \exp [i A \omega \sin (\omega z)] u_{n}^{*} u_{n+1}+\text { H.c. }\right\},
$$

with $2 N$ being the total number of optical waveguides. Chiral symmetry is represented by the sublattice operator $\Gamma=\sum_{n}^{N} u_{2 n-1}^{*} u_{2 n-1}-\sum_{n}^{N} u_{2 n}^{*} u_{2 n}$, which is unitary, Hermitian, and local. Obviously, $\Gamma H \Gamma=-H$; this means that this periodically modulated SSH-like Hamiltonian has chiral symmetry [34]. On the other hand, the above Hamiltonian also has time reversal symmetry, i.e., it is invariant under the transformation $[z \rightarrow-z, i \rightarrow-i]$.

\section{FLOQUET ENERGY SPECTRUM}

Since the system is invariant under $z \rightarrow z+T$, according to the Floquet theorem [20], the steady states of the coupled-mode equation (2) follow

$$
u_{n}(z)=e^{-i E z} \sum_{\chi=-\infty}^{+\infty} e^{-i \chi \omega z} c_{n, \chi},
$$

where $c_{n, \chi}$ is the amplitude of the $\chi$ th Floquet state. Substituting the above Floquet expansion into the coupled-mode equations, one obtains the quasienergy equation in the Floquet 


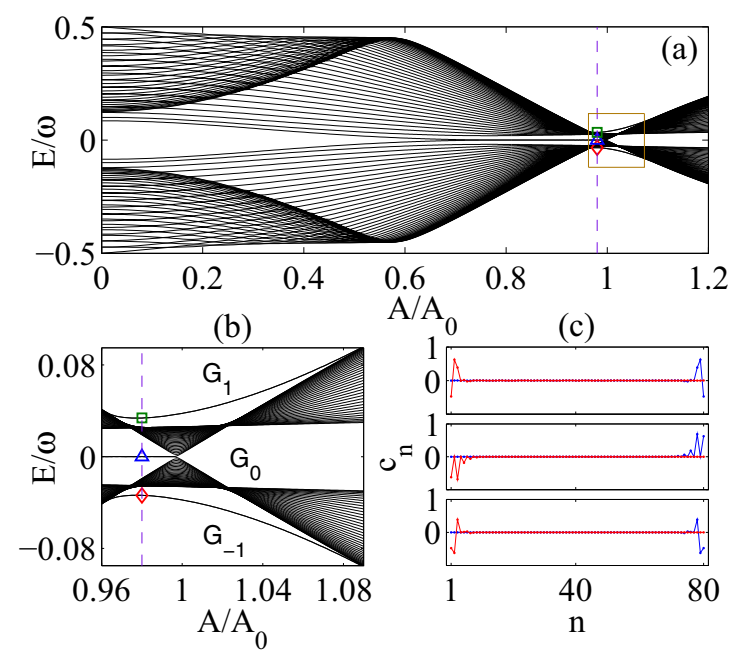

FIG. 2. Quasienergy spectra under open boundary condition. (a) Scaled quasienergy $E / \omega$ vs the scaled modulation amplitude $A / A_{0}$. (b) Enlarged rectangular region of (a). (c) The Floquet edge states corresponding to the square, triangle, and diamond points in the three gaps $\left(G_{+1}, G_{0}, G_{-1}\right)$ at $A / A_{0}=0.98$ marked in (b). The parameters are chosen as $\tau_{1} / \tau_{2}=1.2,2 \pi / \omega=3, A_{0} \omega \simeq 2.405$ [which gives $\left.J_{0}\left(A_{0} \omega\right)=0\right]$, the total lattice number $2 N=80$, and the truncation number $Y=13$.

space:

$$
\begin{aligned}
E c_{n, \chi}= & \sum_{\chi^{\prime}=-\infty}^{+\infty} \tau_{n-1} e^{-i \eta A \omega \sin (\omega z)} e^{-i\left(\chi^{\prime}-\chi\right) \omega z} c_{n-1, \chi^{\prime}} \\
& +\sum_{\chi^{\prime}=-\infty}^{+\infty} \tau_{n} e^{i \eta A \omega \sin (\omega z)} e^{-i\left(\chi^{\prime}-\chi\right) \omega z} c_{n+1, \chi^{\prime}} \\
& +\sum_{\chi^{\prime}=-\infty}^{+\infty} \chi^{\prime} \omega e^{-i\left(\chi^{\prime}-\chi\right) \omega z} c_{n, \chi^{\prime}} \\
& +\sum_{\chi^{\prime} \neq \chi} e^{-i\left(\chi^{\prime}-\chi\right) \omega z} E c_{n, \chi^{\prime}} .
\end{aligned}
$$

We introduce the average over one modulation period for all $z$-dependent quantities and obtain the quasienergy eigenmode equation

$$
\begin{aligned}
E c_{n, \chi}= & \sum_{\chi^{\prime}=-\infty}^{+\infty} \tau_{n-1} J_{\chi-\chi^{\prime}} c_{n-1, \chi^{\prime}} \\
& +\sum_{\chi^{\prime}=-\infty}^{+\infty} \tau_{n} J_{\chi^{\prime}-\chi} c_{n+1, \chi^{\prime}}+\chi \omega c_{n, \chi},
\end{aligned}
$$

where $J_{\chi^{\prime}-\chi}$ is the Bessel function $J_{\chi^{\prime}-\chi}(A \omega)$. To obtain the quasienergy spectrum, one needs to truncate the Floquet space. In our calculation, we choose $\chi^{\prime}, \chi \in[-X, X]$ and $Y=2 X+$ 1 is the truncation number.

Now we discuss the quasienergy spectra under OBC. In Figs. 2(a) and 2(b), we show the scaled quasienergy $E / \omega$ versus the scaled modulation amplitude $A / A_{0}$. In our calculation, $A_{0}$ is given by the first zero point of $J_{0}\left(A_{0} \omega\right), \tau_{1} / \tau_{2}=1.2$, $2 \pi / \omega=3$, and the total lattice number $2 N=80$. In the energy gap $G_{0}$, there appear isolated zero-energy levels under some parameter ranges. Because the quasienergies have periodicity in Floquet space, similar isolated levels can also appear in gaps $G_{ \pm 2, \pm 4, \ldots}$. In the energy gaps $G_{-1}$ and $G_{1}$, isolated nonzero-energy levels appear around $A / A_{0} \sim 1$ and the similar isolated levels can also appear in gap $G_{ \pm 3, \pm 5, \ldots}$. Below, we concentrate our discussion on the quasienergy ranges $-1 / 2 \leqslant$ $E / \omega \leqslant 1 / 2$. In particular, isolated zero- and nonzero-energy levels can coexist in the same parametric region; see Fig. 2(b). The eigenstate profiles, which localize at two edges, indicate that these isolated levels are FESs [see Fig. 2(c)]. We know that the topological edge states in a static SSH model always appear as zero-energy modes. However, in our modulated system, there appear both zero- and nonzero-energy edge states. Naturally, there arises an open question: are all FESs induced by topology?

\section{MULTISCALE ANALYSIS}

To understand how FESs appear in the high-frequency limit, we employ the multiscale perturbation analysis [31,35]. We rewrite Eq. (2) as

$$
-i \frac{d u_{n}}{d z}=\sum_{m} W(z ; n, m) u_{m},
$$

with

$$
\begin{aligned}
W(z ; n, m)= & \frac{1+(-1)^{n}}{2}\left[\delta_{n, m+1} \tau_{1} e^{-i A \omega \sin (\omega z)}\right. \\
& \left.+\delta_{n, m-1} \tau_{2} e^{i A \omega \sin (\omega z)}\right] \\
& +\frac{1-(-1)^{n}}{2}\left[\delta_{n, m+1} \tau_{2} e^{-i A \omega \sin (\omega z)}\right. \\
& \left.+\delta_{n, m-1} \tau_{1} e^{i A \omega \sin (\omega z)}\right] .
\end{aligned}
$$

For the open boundary condition, we have $u_{n<1} \equiv 0$ and $u_{n>2 N} \equiv 0$, in which $2 N$ is the total lattice number. Therefore, $W(z ; n, m)$ can be rewritten as

$$
\begin{aligned}
W(z ; n, m)= & \frac{1+(-1)^{n}}{2}\left[\delta_{n, m+1} \tau_{1} e^{-i A \omega \sin (\omega z)}\right. \\
& \left.+\left(1-\delta_{n, 2 N}\right) \delta_{n, m-1} \tau_{2} e^{i A \omega \sin (\omega z)}\right] \\
& +\frac{1-(-1)^{n}}{2}\left[\left(1-\delta_{n, 1}\right) \delta_{n, m+1} \tau_{2} e^{-i A \omega \sin (\omega z)}\right. \\
& \left.+\delta_{n, m-1} \tau_{1} e^{i A \omega \sin (\omega z)}\right]
\end{aligned}
$$

Because the waveguide axes are periodically curved along the longitudinal propagation ( $z$ direction), we have $W(z ; n, m)=$ $W(z+T ; n, m)$, where $T=2 \pi / \omega$. In the high-frequency limit $(\omega \gg 1)$, we can introduce a small parameter $\varepsilon$, which satisfies $T=O(\varepsilon)$. Thus the solution of Eq. (5) can be given as the series expansion

$$
\begin{aligned}
u_{n}(z)= & U_{n}\left(z_{0}, z_{1}, z_{2}, \ldots\right)+\varepsilon v_{n}\left(z_{-1}, z_{0}, z_{1}, z_{2}, \ldots\right) \\
& +\varepsilon^{2} w_{n}\left(z_{-1}, z_{0}, z_{1}, z_{2}, \ldots\right) \\
& +\varepsilon^{3} \zeta_{n}\left(z_{-1}, z_{0}, z_{1}, z_{2}, \ldots\right)+O\left(\varepsilon^{4}\right)
\end{aligned}
$$


where $z_{l^{\prime}}=\varepsilon^{l^{\prime}} z$. Then the differentiation is performed according to the usual convention:

$$
\frac{d}{d z}=\varepsilon^{-1} \frac{\partial}{\partial z_{-1}}+\frac{\partial}{\partial z_{0}}+\varepsilon \frac{\partial}{\partial z_{1}}+\varepsilon^{2} \frac{\partial}{\partial z_{2}}+\cdots .
$$

In the series solution, the function $U_{n}$ describes the averaged behavior

$$
\left\langle u_{n}\right\rangle=U_{n}, \quad\left\langle\frac{d u_{n}}{d z}\right\rangle=\frac{d U_{n}}{d z},
$$

in which the average notation

$$
\langle\bullet\rangle=\varepsilon T^{-1} \int_{\varepsilon^{-1} z}^{\varepsilon^{-1}(z+T)}(\bullet)\left(z_{-1}\right) d z_{-1} .
$$

It is worth noting that $U_{n}$ does not depend on the "fast" variable $z_{-1}$; this means that

$$
\left\langle U_{n}\right\rangle=U_{n}, \quad\left\langle\frac{d U_{n}}{d z}\right\rangle=\frac{d U_{n}}{d z} .
$$

From Eqs. (9) and (10), we have

$$
\begin{aligned}
\left\langle v_{n}\right\rangle & =\left\langle w_{n}\right\rangle=\left\langle\zeta_{n}\right\rangle \equiv 0, \\
\left\langle\frac{\partial v_{n}}{\partial z_{l^{\prime}}}\right\rangle & =\left\langle\frac{\partial w_{n}}{\partial z_{l^{\prime}}}\right\rangle=\left\langle\frac{\partial \zeta_{n}}{\partial z_{l^{\prime}}}\right\rangle \equiv 0,
\end{aligned}
$$

for $l^{\prime}=-1,0,1,2, \ldots$.

Substituting Eq. (7) into Eq. (5) and collecting terms with different orders of $\varepsilon$, we obtain

$$
-i \frac{\partial U_{n}}{\partial z_{0}}=i \frac{\partial v_{n}}{\partial z_{-1}}+\sum_{m} W(z ; n, m) U_{m},
$$

for the order $\varepsilon^{0}$. Using the conditions Eq. (10) and Eq. (11) and averaging Eq. (12), we have

$$
-i \frac{\partial U_{n}}{\partial z_{0}}=\sum_{m} W_{0}(n, m) U_{m}
$$

where $W_{0}(n, m)=\langle W(z ; n, m)\rangle$. Then substituting Eq. (13) into Eq. (12), we can obtain the equation for $v_{n}$

$$
-i \frac{\partial v_{n}}{\partial z_{-1}}=\sum_{m}\left[W(z ; n, m)-W_{0}(n, m)\right] U_{m} .
$$

Thus through integrating the above equation, we derive an explicit expression for the function $v_{n}$

$$
v_{n}=i \varepsilon^{-1} \sum_{m} M(z ; n, m) U_{m},
$$

with $M(z ; n, m)=\int\left[W(z ; n, m)-W_{0}(n, m)\right] d z$. Here, the function $M$ is periodic and has average zero value

$$
M(z ; n, m) \equiv M(z+T ; n, m) ;\langle M(z ; n, m)\rangle=0 .
$$

For the order $\varepsilon^{1}$, we have

$$
-i \frac{\partial U_{n}}{\partial z_{1}}=i \frac{\partial v_{n}}{\partial z_{0}}+i \frac{\partial w_{n}}{\partial z_{-1}}+\sum_{m} W(z ; n, m) v_{m} .
$$

Substituting Eqs. (15) and (13) into Eq. (17), we obtain

$$
\begin{aligned}
-i \frac{\partial U_{n}}{\partial z_{1}}= & -i \varepsilon^{-1} \sum_{m, j} M(z ; n, j) W_{0}(j, m) U_{m}+i \frac{\partial w_{n}}{\partial z_{-1}} \\
& +i \varepsilon^{-1} \sum_{m, j} W(z ; n, j) M(z ; j, m) U_{m} .
\end{aligned}
$$

Using the conditions (10), (11), and (16) and averaging Eq. (18), we have

$$
-i \frac{\partial U_{n}}{\partial z_{1}}=i \varepsilon^{-1} \sum_{m, j}\langle W(z ; n, j) M(z ; j, m)\rangle U_{m} .
$$

Substituting Eq. (19) into Eq. (18), we can obtain the equation for $w_{n}$ :

$$
\begin{aligned}
-i \frac{\partial w_{n}}{\partial z_{-1}}= & -i \varepsilon^{-1} \sum_{m, j} M(z ; n, j) W_{0}(j, m) U_{m} \\
& +i \varepsilon^{-1} \sum_{m, j}[W(z ; n, j) M(z ; j, m) \\
& -\langle W(z ; n, j) M(z ; j, m)\rangle] U_{m} .
\end{aligned}
$$

Similarly, by performing integration, we can derive the explicit expression for $w_{n}$.

For the order $\varepsilon^{2}$, we have

$$
\begin{aligned}
-i \frac{\partial U_{n}}{\partial z_{2}}= & i \frac{\partial v_{n}}{\partial z_{1}}+i \frac{\partial w_{n}}{\partial z_{0}}+i \frac{\partial \zeta_{n}}{\partial z_{-1}} \\
& +\sum_{m} W(z ; n, m) w_{m}
\end{aligned}
$$

Using Eqs. (10) and (11) and averaging Eq. (21), we obtain

$$
-i \frac{\partial U_{n}}{\partial z_{2}}=\sum_{q}\left\langle W(z ; n, q) w_{q}\right\rangle,
$$

where the second term

$$
\begin{aligned}
\left\langle W(z ; n, q) w_{q}\right\rangle & =\left\langle\left[W(z ; n, q)-W_{0}(n, q)\right] w_{q}\right\rangle \\
& =-\varepsilon^{-1}\left\langle M(z ; n, q) \frac{\partial w_{q}}{\partial_{-1}}\right\rangle .
\end{aligned}
$$

Then using Eqs. (20) and (11), we can rewrite Eq. (22) as

$$
\begin{aligned}
-i \frac{\partial U_{n}}{\partial z_{2}}= & \varepsilon^{-2} \sum_{q, m, j}\langle M(z ; n, q)[W(z ; q, j) \\
& \left.\left.-W_{0}(q, j)\right] M(z: j, m)\right\rangle U_{m} \\
& +\varepsilon^{-2} \sum_{q, m, j}\left\langleM ( z ; n , q ) \left[ W_{0}(q, j) M(Z ; j, m)\right.\right. \\
& \left.\left.-M(z ; q, j) W_{0}(j, m)\right]\right\rangle U_{m} .
\end{aligned}
$$

By combining Eqs. (13), (19), and (24) and using Eq. (8), we obtain a closed-form equation for $U_{n}$ :

$$
-i \frac{d U_{n}}{d z}=\sum_{m} W_{s}(n, m) U_{m} .
$$

Here the effective coupling coefficients are given as

$$
\begin{aligned}
W_{s}(n, m)= & W_{0}(n, m)+\sum_{j} W_{1}(n, j, m) \\
& +\sum_{q, j} W_{2}(n, q, j, m),
\end{aligned}
$$


with

$$
\begin{aligned}
W_{0}(n, m)= & \langle W(z ; n, m)\rangle=\frac{1+(-1)^{n}}{2}\left[\delta_{n, m+1} \tau_{1}\right. \\
& \left.+\left(1-\delta_{n, 2 N}\right) \delta_{n, m-1} \tau_{2}\right] J_{0}(\eta A \omega) \\
& +\frac{1-(-1)^{n}}{2}\left[\left(1-\delta_{n, 1}\right) \delta_{n, m+1} \tau_{2}\right. \\
& \left.+\delta_{n, m-1} \tau_{1}\right] J_{0}(\eta A \omega),
\end{aligned}
$$

$$
\begin{aligned}
\sum_{j} W_{1}(n, j, m)= & i \sum_{j}\langle W(z ; n, j) M(z ; j, m)\rangle=0, \\
\sum_{q, j} W_{2}(n, q, j, m)= & \sum_{q, j}\langle M(z ; n, q)[W(z ; q, j) \\
& \left.\left.-W_{0}(q, j)\right] M(z ; j, m)\right\rangle \\
& +\sum_{q, j}\left\langleM ( z ; n , q ) \left[ W_{0}(q, j) M(z ; j, m)\right.\right. \\
& \left.\left.-M(z ; q, j) W_{0}(j, m)\right]\right\rangle \\
= & \frac{1+(-1)^{n}}{2}\left\{\delta_{n, m+1}\left[\left(\tau_{1} / \tau_{2}\right)^{2}-1\right]\left(\tau_{1} / \tau_{2}\right)\right. \\
& \left.+\delta_{n, m-1}\left[1-\left(\tau_{1} / \tau_{2}\right)^{2}\right]\right\} \Delta \\
& +\frac{1-(-1)^{n}}{2}\left\{\delta_{n, m+1}\left[1-\left(\tau_{1} / \tau_{2}\right)^{2}\right]\right. \\
& \left.+\delta_{n, m-1}\left[\left(\tau_{1} / \tau_{2}\right)^{2}-1\right]\left(\tau_{1} / \tau_{2}\right)\right\} \Delta \\
& +\frac{\tau_{1}}{2 \tau_{2}}\left(\delta_{n, 1} \delta_{m, 2}+\delta_{n, 2} \delta_{m, 1}\right. \\
& \left.+\delta_{n, 2 N} \delta_{m, 2 N-1}+\delta_{n, 2 N-1} \delta_{m, 2 N}\right) \Delta,
\end{aligned}
$$

with

$$
\begin{aligned}
\Delta= & -\omega^{-2} \tau_{2}^{3} \sum_{m \neq 0} \sum_{j \neq 0,-m} J_{j}(A \omega) \\
& \times J_{m}(A \omega) J_{j+m}(A \omega) j^{-1} m^{-1} .
\end{aligned}
$$

Finally, the effective equations for the slowly varying functions $U_{n}(z)$ read as

$$
\begin{aligned}
-i \frac{d U_{2 n-1}}{d z}= & \tau_{a} U_{2 n}+\tau_{b} U_{2 n-2}+\delta_{(2 n-1,1)} \tau_{c} U_{2} \\
& +\delta_{(2 n-1,2 N-1)} \tau_{c} U_{2 N}, \\
-i \frac{d U_{2 n}}{d z}= & \tau_{b} U_{2 n+1}+\tau_{a} U_{2 n-1}+\delta_{(2 n, 2)} \tau_{c} U_{1} \\
& +\delta_{(2 n, 2 N)} \tau_{c} U_{2 N-1},
\end{aligned}
$$

with Kronecker's delta function $\delta_{(n, m)}$. Here, the effective couplings are given as

$$
\begin{aligned}
\tau_{a} & =\tau_{1} J_{0}-\left(\tau_{1} / \tau_{2}\right) \Theta, \\
\tau_{b} & =\tau_{2} J_{0}+\Theta, \\
\tau_{c} & =\tau_{1} \Delta /\left(2 \tau_{2}\right),
\end{aligned}
$$

with $\Delta=-\omega^{-2} \tau_{2}^{3} \sum_{m \neq 0} \sum_{j \neq\{0,-m\}} J_{j} J_{m} J_{j+m} j^{-1} m^{-1}$ and $\Theta=$ $\left[1-\left(\tau_{1} / \tau_{2}\right)^{2}\right] \Delta$. The effective couplings $\tau_{c}$ describe the virtual defects at boundaries, as shown in the schematic diagram in Fig. 4.
Based on the above discussions, the periodically modulated system can be described by effective static SSH-like coupledmode Eqs. (27). The major difference is the existence of virtual defects at boundaries in the effective model. Similar to a surface perturbation, the virtual defects can form defect-free surface states (or FESs) [31]. On the other hand, if $\tau_{c}=0$, the static SSH-like coupled-mode equations reduce to the conventional SSH model [33] and the defect-free surface states disappear. However, the 1D conventional SSH model belonging to the BDI symmetry class [36], which satisfies time reversal and chiral symmetry, can support an $Z$ topological index (the integer $Z$ index can only take values zero or 1) [37]. For $\left|\tau_{a}\right| /\left|\tau_{b}\right|<1$, this system is topologically nontrivial and has one zero-energy mode localized at each edge - the zero-energy edge mode also called Shockley-like surface states [38]. For $\left|\tau_{a}\right| /\left|\tau_{b}\right|>1$, the system is topologically trivial with no edge modes. If change $\tau_{c} \neq 0$, the static SSH-like coupled-mode equations still satisfy time reversal and chiral symmetry, which illustrates that the multiscale perturbation analysis does not change the symmetry of the system. Similar to the static system, the relation between Shockley-like and Tamm-like surface states has been discussed in [38-40]. Their results show that the transitions between Shockley-like and Tamm-like surface states are observed by tuning the surface perturbation (embedded defects). In our system, without any embedded or nonlinearity-induced defects, the surface perturbation (virtual defects) is induced by periodical modulations. In the next section, we will give the parameter regions of FESs and explore their topological nature.

\section{NONTOPOLOGICAL VS TOPOLOGICAL EDGE STATES}

\section{A. Asymptotic phase boundary}

To estimate the cutoff values (phase boundaries) for the regions of FESs caused by virtual defects. We now consider stationary solutions in the form of $U_{n}(z)=U_{n}(0) e^{i E z}$ with $E$ being the propagation constant. Substituting it into Eq. (27), we obtain

$$
\begin{aligned}
E U_{2 n-1}= & \tau_{a} U_{2 n}+\tau_{b} U_{2 n-2} \\
& +\left(\delta_{2 n-1,1} \tau_{c} U_{2}+\delta_{2 n-1,2 N-1} \tau_{c} U_{2 N}\right) \\
E U_{2 n}= & \tau_{b} U_{2 n+1}+\tau_{a} U_{2 n-1} \\
& +\left(\delta_{2 n, 2} \tau_{c} U_{1}+\delta_{2 n, 2 N} \tau_{c} U_{2 N-1}\right)
\end{aligned}
$$

For an infinite lattice, we have

$$
\begin{aligned}
E U_{2 n-1} & =\tau_{a} U_{2 n}+\tau_{b} U_{2 n-2}, \\
E U_{2 n} & =\tau_{b} U_{2 n+1}+\tau_{a} U_{2 n-1} .
\end{aligned}
$$

The solution of Eqs. (30) can be given as the ansatz

$$
\begin{aligned}
U_{2 n-1} & =a_{1} Q e^{i k n}+a_{2} P e^{-i k n}, \\
U_{2 n} & =a_{1} P e^{i k n}+a_{2} Q e^{-i k n},
\end{aligned}
$$

where $a_{1}$ and $a_{2}$ are arbitrary nonzero constants. Substituting Eqs. (31) into Eqs. (30), we obtain

$$
E\left[\begin{array}{l}
P \\
Q
\end{array}\right]=\left[\begin{array}{cc}
0 & \tau_{a}+\tau_{b} e^{i k} \\
\tau_{a}+\tau_{b} e^{-i k} & 0
\end{array}\right]\left[\begin{array}{l}
P \\
Q
\end{array}\right] .
$$


Then we can have

$$
\frac{P}{Q}=\frac{E}{\tau_{a}+\tau_{b} e^{-i k}}=\frac{\tau_{a}+\tau_{b} e^{i k}}{E} .
$$

Therefore, the propagation constant is given as

$$
E^{2}=\tau_{a}^{2}+\tau_{b}^{2}+2 \tau_{a} \tau_{b} \cos (k),
$$

for $k \in[-\pi, \pi]$.

For a finite but sufficiently large number of lattices $(2 \mathrm{~N}=$ 80 in our calculation), considering the two edges, we have

$$
\begin{aligned}
E U_{2} & =\left(\tau_{a}+\tau_{c}\right) U_{1}+\tau_{b} U_{3}, \\
E U_{1} & =\left(\tau_{a}+\tau_{c}\right) U_{2}, \\
E U_{2 N} & =\left(\tau_{a}+\tau_{c}\right) U_{2 N-1}, \\
E U_{2 N-1} & =\left(\tau_{a}+\tau_{c}\right) U_{2 N}+\tau_{b} U_{2 N-2} .
\end{aligned}
$$

Besides $U_{1}$ and $U_{2 N}$, the coupling equations are consistent with Eqs. (30). So that we should rewrite the ansatz, similar to Eqs. (31), we have

$$
\begin{aligned}
U_{2 n-1} & =U_{1} \quad(n=1), \\
U_{2 n-1} & =a_{1} Q e^{i k n}+a_{2} P e^{-i k n} \quad(1<n \leqslant N), \\
U_{2 n} & =a_{1} P e^{i k n}+a_{2} Q e^{-i k n} \quad(1 \leqslant n<N), \\
U_{2 n} & =U_{2 N} \quad(n=N) .
\end{aligned}
$$

First, we consider the left boundary of lattices and we can give a set of equations

$$
\begin{aligned}
E U_{2} & =\left(\tau_{a}+\tau_{c}\right) U_{1}+\tau_{b} U_{3}, \\
E U_{1} & =\left(\tau_{a}+\tau_{c}\right) U_{2}, \\
E U_{2(N-1)} & =\tau_{a} U_{2(N-1)-1}+\tau_{b} U_{2(N-1)+1} .
\end{aligned}
$$

Combining Eqs. (36) and Eqs. (37), we have

$$
\begin{aligned}
& \frac{e^{-i k 2(N-1)}}{e^{i k 2(N-1)}} \\
& =\frac{\left[\tau_{b} \frac{P}{Q} e^{-i k}+\frac{\left(\tau_{a}+\tau_{c}\right)^{2}}{E}-E\right]\left(E \frac{P}{Q}-\tau_{b} e^{i k}-\tau_{a} e^{-i k}\right)}{\left[E \frac{P}{Q}-\tau_{b} e^{i k}-\frac{\left(\tau_{a}+\tau_{c}\right)^{2}}{E} \frac{P}{Q}\right]\left(\tau_{b} \frac{P}{Q} e^{-i k}-E+\tau_{a} \frac{P}{Q} e^{i k}\right)} .
\end{aligned}
$$

We set $k=-i \varrho$ and have $\frac{e^{-i k 2(N-1)}}{e^{i k 2(N-1)}}=e^{-4 \varrho(N-1)}$, where $\varrho$ is a real number. If $\varrho>0$, when $N \rightarrow \infty$ we have $e^{-4 \varrho(N-1)} \simeq 0$ and equivalent to

$$
\left[\tau_{b} \frac{P}{Q} e^{-\varrho}+\frac{\left(\tau_{a}+\tau_{c}\right)^{2}}{E}-E\right]\left(E \frac{P}{Q}-\tau_{b} e^{\varrho}-\tau_{a} e^{-\varrho}\right) \simeq 0 .
$$

If $\varrho<0$, when $N \rightarrow \infty$ we have $e^{-4 \varrho(N-1)} \simeq \infty$ and equivalent to

$$
\begin{aligned}
& {\left[E \frac{P}{Q}-\tau_{b} e^{\varrho}-\frac{\left(\tau_{a}+\tau_{c}\right)^{2}}{E} \frac{P}{Q}\right]} \\
& \times\left(\tau_{b} \frac{P}{Q} e^{-\varrho}-E+\tau_{a} \frac{P}{Q} e^{\varrho}\right) \simeq 0 .
\end{aligned}
$$

Combining Eq. (33) and Eq. (39), we have

$$
e^{\varrho}=\frac{\tau_{c}\left(\tau_{c}+2 \tau_{a}\right)}{\tau_{a} \tau_{b}}=e^{i k}=d .
$$

Similarly, combining Eq. (33) and Eq. (40), we have

$$
e^{-\varrho}=\frac{\tau_{a} \tau_{b}}{\tau_{c}\left(\tau_{c}+2 \tau_{a}\right)}=e^{-i k}=d^{-1} .
$$

Thus in the vicinity of the self-collimation point $\left[J_{0}\left(A_{0} \omega\right)=\right.$ $0]$, as the couplings $\left(\tau_{a}, \tau_{b}\right)$ are very weak, the edge states induced by the virtual defects with the quasienergies $E_{s}$ are given as

$$
\begin{aligned}
E_{s}^{2} & =\tau_{a}^{2}+\tau_{b}^{2}+\tau_{a} \tau_{b}\left[e^{i k}+e^{-i k}\right] \\
& =\tau_{a}^{2}+\tau_{b}^{2}+\tau_{a} \tau_{b}\left[d+d^{-1}\right] .
\end{aligned}
$$

On the other hand, when we consider the right boundary of lattices, we can also obtain the surface energy $E_{s}$ which is in agreement with Eq. (43).

Obviously, when $E_{s}^{2}>\max \left(E^{2}\right)$, FESs appear in the energy gaps $G_{-1}$ and $G_{1}$. Otherwise, when $E_{s}^{2}<\min \left(E^{2}\right)$, FESs appear in the gap $G_{0}$. Obviously, $\max \left(E^{2}\right)$ and $\min \left(E^{2}\right)$ are given by $|\cos (k)|=1$. From $\cos (k)=+1$, one can obtain the cutoff values

$$
A_{c s}^{1,2} / A_{0} \simeq 1-\frac{\tau_{1} \tilde{\tau}_{c} \pm F_{a}}{\tau_{1} \tau_{2}} .
$$

From $\cos (k)=-1$, one can obtain the cutoff values

$$
A_{c s}^{3,4} / A_{0} \simeq 1-\frac{-\tau_{1} \tilde{\tau_{c}} \pm F_{b}}{\tau_{1} \tau_{2}} .
$$

Here, $A_{0}$ is the first root of the Bessel function $J_{0}(A \omega)=0$, $F_{a}=\sqrt{\left(\tau_{1} \tilde{\tau}_{c}\right)^{2}+\tau_{1} \tau_{2} M_{+}}, \quad F_{b}=\sqrt{\left(\tau_{1} \tilde{\tau}_{c}\right)^{2}+\tau_{1} \tau_{2} M_{-}}, \quad \tilde{\tau}_{c}=$ $\frac{\tau_{1}}{2 \tau_{2}} \tilde{\Delta}, \quad M_{ \pm}=\frac{\tau_{1}}{\tau_{2}}\left[1-\left(\frac{\tau_{1}}{\tau_{2}}\right)^{2}\right] \tilde{\Delta}\left\{\left[1-\left(\frac{\tau_{1}}{\tau_{2}}\right)^{2}\right] \tilde{\Delta} \mp 2 \tilde{\tau}_{c}\right\} \pm\left(\tilde{\tau}_{c}\right)^{2}$, and $\tilde{\Delta}=\left.\Delta\right|_{A \rightarrow A_{0}}$. These cutoff values define the boundaries between the regions with and without FESs; see the dashed blue curves in Figs. 4(a) and 4(b), which are also called defect-free surface states [31]. Since $F_{b}$ is a purely imaginary number for all $2 \pi / \omega$ when $\tau_{1} / \tau_{2}=1.2$, in Fig. 4(a), there are no cutoff values $A_{c s}^{3,4} / A_{0}$. When $2 \pi / \omega \rightarrow 0$, all cutoff values gradually converge into one point at $A / A_{0}=1$, and there are no FESs caused by the virtual defects.

On the other hand, as the effective model Eq. (27) is an SSH-like model, the system changes from topological to nontopological when the effective coupling is tuned from $\left|\tau_{a}\right|<$ $\left|\tau_{b}\right|$ to $\left|\tau_{a}\right|>\left|\tau_{b}\right|$. The effective couplings $\left(\tau_{a}, \tau_{b}\right)$ depend on the original couplings $\left(\tau_{1}, \tau_{2}\right)$ and the driving parameters $(A, \omega)$. We show the effective coupling strengths $\left(\left|\tau_{a}\right|,\left|\tau_{b}\right|\right)$ versus the scaled modulation amplitude $A / A_{0}$ for $2 \pi / \omega=2$ and $\tau_{1} / \tau_{2}=1.2$; see the inset in Fig. 4(a). There appear two intersection points at $\left|\tau_{a}\right|=\left|\tau_{b}\right|$ when $A / A_{0}$ increases. In the regions of $\left|\tau_{a}\right|<\left|\tau_{b}\right|$, topological FESs appear (the relevant cumulative phase being $\pi$ ), which is also called Shockley-like surface states [38]. The intersection points, where topological phase transition points occur, are given by

$$
A_{c t}^{5,6} / A_{0} \simeq 1+\frac{\left(1 \pm \tau_{1} / \tau_{2}\right)^{2} \tilde{\Delta}}{\tau_{2}} .
$$

See the dashed blue curves 5 and 6 in the inset of Fig. 4(b). Similarly, when $2 \pi / \omega \rightarrow 0$, these two curves also gradually converge into one point at $A / A_{0}=1$. Thus, in the limit of $2 \pi / \omega=0$, the effective couplings vanish when $A / A_{0}=1$ and the modulation does not change the topological feature when $A / A_{0}$ is tuned through $A / A_{0}=1$. 


\section{B. Zak phase}

To distinguish topological and nontopological FESs, we calculate the bulk topological invariant-the Zak phase [41]. The Zak phase can predict the existence (with the relevant cumulative phase being $\pi$ ) or absence (vanishing cumulative phase) of topological FESs in specific gaps.

For a modulated SSH system of $N$ cells (i.e., $2 N$ lattices) under PBC, by implementing a Fourier transform

$$
\begin{aligned}
c_{2 n-1, \chi} & =\frac{1}{\sqrt{N}} \sum_{k} e^{i k(2 n-1)} c_{1, k, \chi}, \\
c_{2 n, \chi} & =\frac{1}{\sqrt{N}} \sum_{k} e^{i k 2 n} c_{2, k, \chi},
\end{aligned}
$$

we obtain the quasienergy spectra and the eigenstates by diagonalizing the quasienergy equation

$$
E_{l}\left(\begin{array}{c}
c_{1, k, \chi}^{(l)} \\
c_{2, k, \chi}^{(l)}
\end{array}\right)=\sum_{\chi^{\prime}} \widehat{\mathcal{R}}(k)\left(\begin{array}{c}
c_{1, k, \chi^{\prime}}^{(l)} \\
c_{2, k, \chi^{\prime}}^{(l)}
\end{array}\right)+\chi \omega \hat{I}\left(\begin{array}{c}
c_{1, k, \chi}^{(l)} \\
c_{2, k, \chi}^{(l)}
\end{array}\right),
$$

with the $2 \times 2$ unit matrix $\hat{I}$ and the matrix

$$
\widehat{\mathcal{R}}(k)=\left(\begin{array}{cc}
0 & P_{F}(k) \\
\tilde{P}_{F}(k) & 0
\end{array}\right) .
$$

Here, $P_{F}=\tau_{1} J_{\chi^{\prime}-\chi} e^{i k}+\tau_{2} J_{\chi-\chi^{\prime}} e^{-i k}, \quad \tilde{P}_{F}=\tau_{1} J_{\chi-\chi^{\prime}} e^{-i k}+$ $\tau_{2} J_{\chi^{\prime}-\chi} e^{i k}$, and $k$ denotes the quasimomentum.

To compute the Zak phase for the Floquet quasienergy spectrum one needs to truncate the Floquet space. The number of replicas needs to be chosen so that all relevant transitions at the desired energy are kept. The Zak phase $Z_{G_{m}}$ for a specific gap is given by summing up $Z^{(l)}$ for all bands below the gap, where $Z^{(l)}=i \oint_{k}\left\langle c_{k}^{(l)}\left|\partial_{k}\right| c_{k}^{(l)}\right\rangle d k$ and the eigenstates $\left|c_{k}^{(l)}\right\rangle=\sum_{\alpha, \chi} c_{\alpha, k, \chi}^{(l)}|\alpha, k, \chi\rangle$ for the $l$ th band are superposition states of different Floquet-Bloch states $|\alpha, k, \chi\rangle$. For a gap between the $(Y+m)$ th and $(Y+m+1)$ th bands, its Zak phase $Z_{G_{m}}$ is defined as

$$
Z_{G_{m}}=\sum_{l=1}^{Y+m} Z^{(l)}=\sum_{l=1}^{Y+m}\left[i \oint_{k}\left\langle c_{k}^{(l)}\left|\partial_{k}\right| c_{k}^{(l)}\right\rangle d k\right] .
$$

For example, the Zak phase $Z_{G_{1}}$ can be calculated by summing up all $Z^{(l)}$ for the bands below the gap $G_{1}$; see in Fig. 3 .

\section{Phase diagram}

To verify the above analytical results, we numerically calculate the quasienergy spectra. From the quasienergy spectra under OBC, we indeed find several FESs appear. We then calculate Zak phases of the corresponding bulk states under PBC and find that the Zak phase $Z_{G_{m}}$ for a specific gap is either zero or $\pi$ and topological FESs only appear in a gap of nonzero $Z_{G_{m}}$.

In Fig. 4, we show the phase diagram of all possible FESs in the parameter plane $\left(2 \pi / \omega, A / A_{0}\right)$. The appearance of topological FESs (red regions) and nontopological FESs (yellow regions) and their coexistence (mesh regions) sensitively depends on the coupling ratio $\tau_{1} / \tau_{2}$ and the modulation parameters $\left(\omega, A / A_{0}\right)$. In the absence of modulation, topological edge states appear only if $\tau_{1} / \tau_{2}<1$; otherwise, no

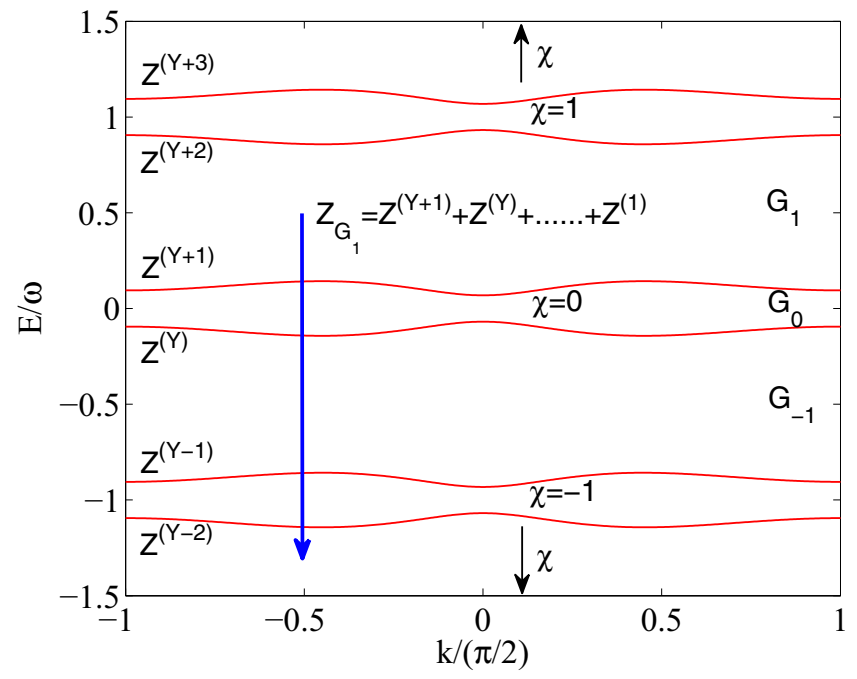

FIG. 3. Quasienergy spectrum in the quasimomentum space and the Zak phase for the gap $G_{1}$.

edge state appears. However, by applying a proper modulation, topological FESs may appear even if $\tau_{1} / \tau_{2}>1$ and also may disappear even if $\tau_{1} / \tau_{2}<1$. In addition to the regions of topological and nontopological FESs, there exists the region of no edge states. When $2 \pi / \omega \rightarrow 0$, topological FESs appear if $\tau_{1} / \tau_{2}<1$ and all nontopological FESs gradually vanish at the zero point of the Bessel function $J_{0}\left(A_{0} \omega\right)=0$. Our numerical results clearly show all phase boundaries (the solid curves) gradually converge into one point at $A / A_{0}=1$ when $2 \pi / \omega \rightarrow 0$, which well agree with our analytical results (the dashed blue curves).

\section{Noncoexistence of nontopological and topological Floquet edge states in the same gap}

Although nontopological and topological FESs can be supported by the same parameters, we find that they cannot appear in the same energy gap. In this section, we only consider the quasienergy ranges $-1 / 2 \leqslant E / \omega \leqslant 1 / 2$, so that the topological FESs (Zak phase $Z_{G_{0}}=\pi$ ) only possibly appear in gap $G_{0}$. We will prove that nontopological and topological FESs cannot coexist in the gap $G_{0}$. For the whole Floquet spaces, due to the periodicity of quasienergy, this proves indirect reflection the topological FESs cannot appear in the gap $G_{ \pm 1, \pm 3, \pm 5, \ldots}$; in addition the nontopological and topological FESs cannot coexist in the gap $G_{0, \pm 2, \pm 4, \ldots}$.

If nontopological FESs appear in the gap $G_{0}$, the edge state quasienergy $E_{s}$ and the bulk-state quasienergy $E$ will satisfy the condition $E_{s}^{2}<\min \left(E^{2}\right)$. From Eq. (34), the condition $E_{s}^{2}<\min \left(E^{2}\right)$ reads

$$
E_{s}^{2}<\min \left(\tau_{a}^{2}+\tau_{b}^{2}-2 \tau_{a} \tau_{b}, \tau_{a}^{2}+\tau_{b}^{2}+2 \tau_{a} \tau_{b}\right),
$$

which requests the parameters obeying $\tau_{c}\left(\tau_{c}+2 \tau_{a}\right)<0$. As the nontopological FESs appear around $A / A_{0} \sim 1$, we have $\tau_{c}=\frac{\tau_{1}}{2 \tau_{2}} \Delta<0$, so that the above inequality is equivalent to

$$
2 \tau_{1} J_{0}(A \omega)-2 \frac{\tau_{1}}{\tau_{2}}\left[1-\left(\frac{\tau_{1}}{\tau_{2}}\right)^{2}\right] \Delta+\frac{1}{2}\left(\frac{\tau_{1}}{\tau_{2}}\right) \Delta>0 .
$$



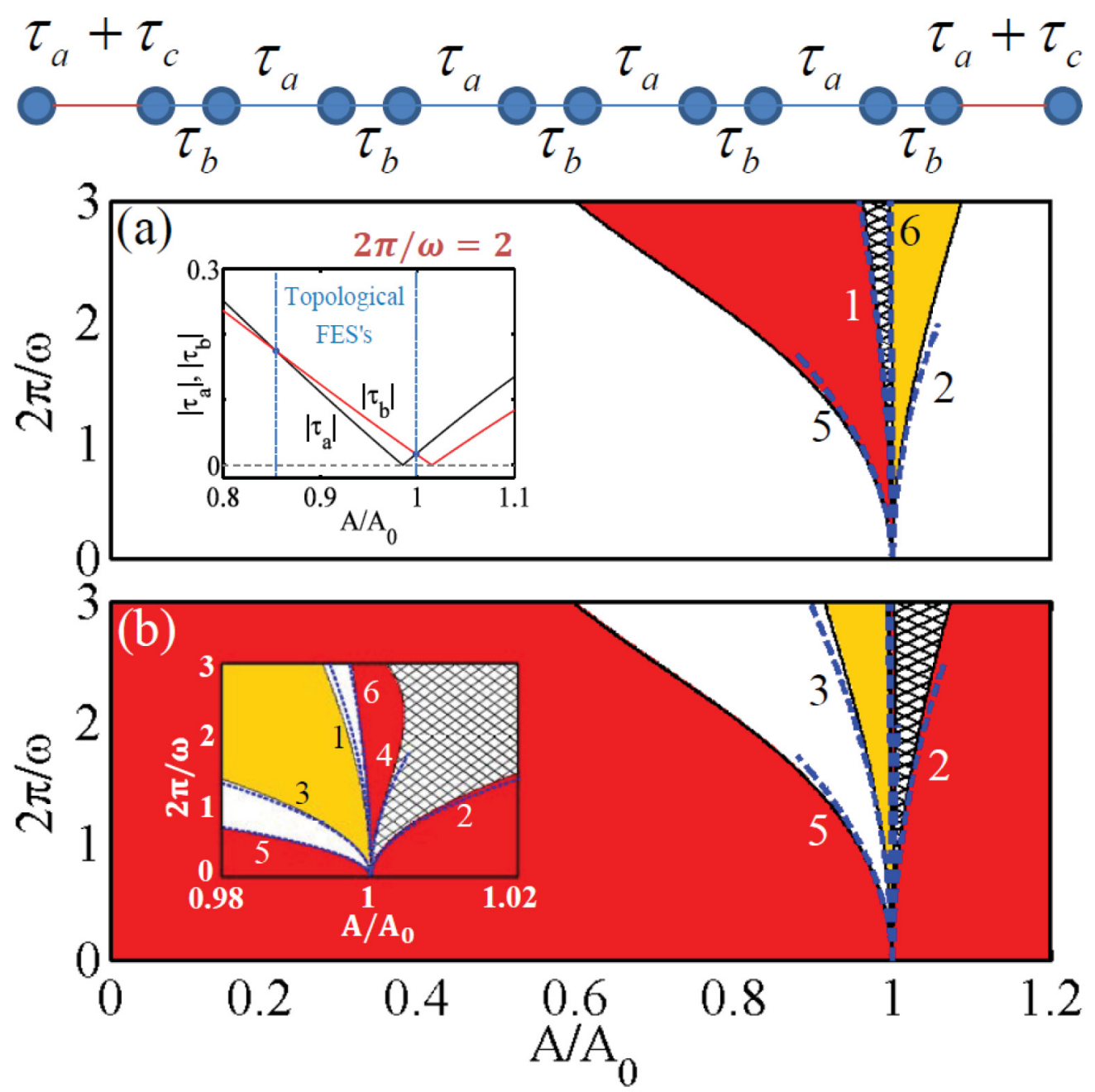

FIG. 4. Phase diagram of the Floquet edge states. Top: schematic diagram for the effective model Eq. (27). (a),(b) Phase diagrams for (a) $\tau_{1} / \tau_{2}=1.2$ and (b) $\tau_{2} / \tau_{1}=1.2$. The red regions only support topological FESs, the yellow regions only support nontopological FESs, and the mesh regions support both topological and nontopological FESs. The curves 1, 2, 3, and 4 respectively correspond to the nontopological FES cutoff values $A_{c s}^{1} / A_{0}, A_{c s}^{2} / A_{0}, A_{c s}^{3} / A_{0}$, and $A_{c s}^{4} / A_{0}$, while the curves 5 and 6 respectively correspond to the topological transition points $A_{c t}^{5} / A_{0}$ and $A_{c t}^{6} / A_{0}$, where the inset in (b) is the enlarged region nearby $A / A_{0} \sim 0$. The system changes from topological to nontopological when the effective couplings are tuned from $\left|\tau_{a}\right|<\left|\tau_{b}\right|$ to $\left|\tau_{a}\right|>\left|\tau_{b}\right|$; see the inset in (a) for $2 \pi / \omega=2$.

Below we separately discuss the two cases: (I) $\tau_{2}>\tau_{1}>0$ and (II) $\tau_{1}>\tau_{2}>0$.

Case I: $\tau_{2}>\tau_{1}>0$. Without loss of generality, one can set $\tau_{2}=1$.

As $\tau_{a}<0$ always contradicts the condition (50), the appearance of nontopological FESs in the gap $G_{0}$ requests

$$
\begin{aligned}
& \tau_{a}=\tau_{1} J_{0}(A \omega)-\tau_{1}\left[1-\left(\tau_{1}\right)^{2}\right] \Delta>0, \\
& \tau_{b}=J_{0}(A \omega)+\left[1-\left(\tau_{1}\right)^{2}\right] \Delta<0, \\
& 2 \tau_{1} J_{0}(A \omega)-2 \tau_{1}\left[1-\left(\tau_{1}\right)^{2}\right] \Delta+\frac{1}{2} \tau_{1} \Delta>0
\end{aligned}
$$

or

$$
\begin{aligned}
& \tau_{a}=\tau_{1} J_{0}(A \omega)-\tau_{1}\left[1-\left(\tau_{1}\right)^{2}\right] \Delta>0, \\
& \tau_{b}=J_{0}(A \omega)+\left[1-\left(\tau_{1}\right)^{2}\right] \Delta>0, \\
& 2 \tau_{1} J_{0}(A \omega)-2 \tau_{1}\left[1-\left(\tau_{1}\right)^{2}\right] \Delta+\frac{1}{2} \tau_{1} \Delta>0 .
\end{aligned}
$$

On the other hand, in the vicinity of $A_{0}$, we have $J_{0}(A \omega)<$ 0 when $A \rightarrow A_{0}^{+}$and $J_{0}(A \omega)>0$ when $A \rightarrow A_{0}^{-}$. Therefore, from the condition (51), one can obtain (C1) $\left(0<\tau_{1}<\right.$ $\sqrt{1-\digamma}) \cap\left(0<\tau_{1}<\sqrt{3 / 4+\digamma}\right)$ when $A \rightarrow A_{0}^{-}$and (C2) $\left(0<\tau_{1}<\sqrt{3 / 4+\digamma}\right)$ when $A \rightarrow A_{0}^{+}$. Here, the parameter $\digamma$ is given as $\digamma=\frac{\omega^{2} J_{0}(A \omega)}{4 J_{1}^{2}(A \omega) J_{2}(A \omega)}$. However, under the condition $(\mathrm{C} 2)$, one can find that $E_{s}^{2}<0$; this means that the condition (C2) does not support nontopological FESs in the gap $G_{0}$. As we always have $\tau_{b}<0$ when $A \rightarrow A_{0}^{+}$, from the condition (52), we drive the condition (C3): $(\sqrt{1-\digamma}<$ $\tau_{1}<\sqrt{3 / 4+\digamma}$ ) when $A \rightarrow A_{0}^{-}$. Therefore, the appearance of nontopological FESs in the gap $G_{0}$ always request $A \rightarrow A_{0}^{-}$ (where $\tau_{a}>0$ ).

As the effective model (27) is a SSH-like model, under the condition of $\left|\tau_{a}\right| /\left|\tau_{b}\right|<1$, the topological FESs are zeroenergy modes and always appear in the gap $G_{0}$. When $A \rightarrow A_{0}^{-}$ (where $\tau_{a}>0$ ), from $\left|\tau_{a}\right| /\left|\tau_{b}\right|<1$, one can obtain (D1) $(0<$ $\left.\tau_{1}<1-\sqrt{\digamma}\right)$ for $\tau_{b}<0$ and (D2) $\left(0<\tau_{1}<\sqrt{\digamma}-1\right)$ for 
(a)

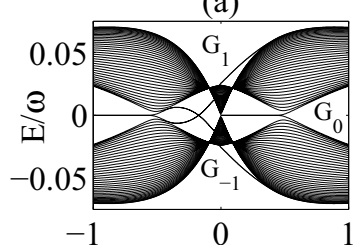

(c)

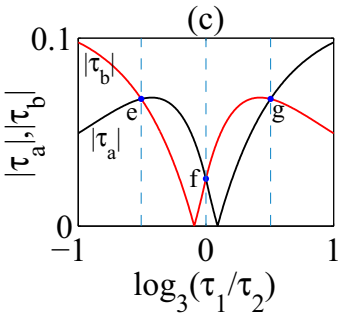$$
1
$$

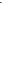

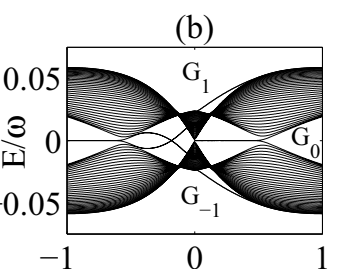

(d)

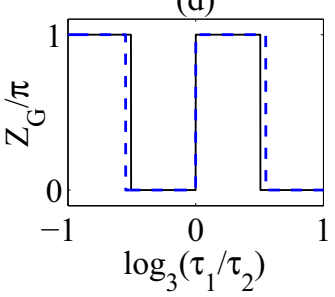

FIG. 5. Scaled quasienergy $E / \omega$ vs coupling ratio $\tau_{1} / \tau_{2}$. (a) Band-gap structure of the effective model (27). (b) Band-gap structure of the original model (2). (c) Effective coupling strengths $\left(\left|\tau_{a}\right|,\left|\tau_{b}\right|\right)$ vs the coupling ratio $\tau_{1} / \tau_{2}$. (d) The Zak phases for the gap $G_{0}$, in which the black and dashed blue lines correspond to the effective and original models, respectively. The parameters are chosen as $A / A_{0}=0.98$, $2 \pi / \omega=3, A_{0} \omega \simeq 2.405$, the total lattice $2 N=80$, and the truncation number $Y=13$.

$\tau_{b}>0$. However, under the conditions (D1) and (D2), one can find that $E_{s}^{2}<0$, which means the absence of nontopological FESs. That is to say, the nontopological and topological FESs cannot coexist in the gap $G_{0}$.

Case II: $\tau_{1}>\tau_{2}>0$. Without loss of generality, one can set $\tau_{1}=1$.

As $\tau_{a}<0$ always contradicts the condition (50), the existence of nontopological FESs in the gap $G_{0}$ requests $\tau_{a}>0$. On the other hand, in the vicinity of $A_{0}$, we have $J_{0}(A \omega)<0$ when $A \rightarrow A_{0}^{+}$and $J_{0}(A \omega)>0$ when $A \rightarrow A_{0}^{-}$. When $\tau_{1}>$ $\tau_{2}>0$, we always have $\tau_{a}<0$ when $A \rightarrow A_{0}^{+}$, so that the appearance of nontopological FESs in the gap $G_{0}$ always requests $A \rightarrow A_{0}^{-}$. Moreover, when $A \rightarrow A_{0}^{-}$, we always have $\tau_{b}=\tau_{2} J_{0}(A \omega)+\left[1-\left(\frac{1}{\tau_{2}}\right)^{2}\right] \Delta>0$. Thus the appearance of nontopological FESs in the gap $G_{0}$ requests

$$
\begin{aligned}
\tau_{a}= & J_{0}(A \omega)-\frac{1}{\tau_{2}}\left[1-\left(\frac{1}{\tau_{2}}\right)^{2}\right] \Delta>0, \\
\tau_{b}= & \tau_{2} J_{0}(A \omega)+\left[1-\left(\frac{1}{\tau_{2}}\right)^{2}\right] \Delta>0, \\
& 2 J_{0}(A \omega)-2 \frac{1}{\tau_{2}}\left[1-\left(\frac{1}{\tau_{2}}\right)^{2}\right] \Delta+\frac{1}{2}\left(\frac{1}{\tau_{2}}\right) \Delta>0 .
\end{aligned}
$$

The above condition (53) requests $\left(\frac{2}{\sqrt{3}} \sqrt{1-\digamma}<\tau_{2}<1\right)$. However, under this condition, one can find that $E_{s}^{2}<0$, which means the absence of nontopological FESs. This means that nontopological FESs cannot appear in gap $G_{0}$ when $\tau_{1}>\tau_{2}>0$ and so there is no coexistence of nontopological and topological FESs.

In order to explore how the ratio $\tau_{1} / \tau_{2}$ affects the FESs, we show how the scaled quasienergy spectrum depends on $\tau_{1} / \tau_{2}$. The quasienergy spectra and Zak phases show that, even when the modulation frequency is not very high, the effective model may well explain the behaviors in the original system. The devi-

ation between the effective and original models decreases with the modulation frequency and gradually vanishes in the highfrequency limit. In Fig. 5, we show the quasienergy spectra, the effective couplings, and Zak phases for $A / A_{0}=0.98$ and $2 \pi / \omega=3$. Although the quasienergies have small differences, the band-gap structures are almost the same, in which both zero and nonzero FESs may appear in different gaps; see Figs. 5(a) and 5(b). From the effective model, topological FESs are always zero-energy modes and only appear in the gap $G_{0}$ when $\left|\tau_{a}\right| /\left|\tau_{b}\right|<1$; see Figs. 5(a) and 5(c). In addition to the topological FESs, due to the modulation-induced virtual defects, there also exist nontopological FESs in different gaps. Moreover, the band-gap structures show that topological and nontopological FESs cannot appear in the same gap, which confirms our previous analytical analysis. From the Zak phases, the effective and original models show similar topological phase transitions, but the transition points show small deviations dependent upon the modulation frequency; see Fig. 5(d).

\section{CONCLUSION}

In summary, we have studied the Floquet edge states in arrays of curved optical waveguides described by the periodically modulated SSH model. According to the Floquet theorem, we give the quasienergy spectra under OBC and find several FESs. To understand how FESs appear, we employ the multiscale perturbation analysis and find the periodic modulations can induce virtual defects at boundaries. Similar to a surface perturbation, the virtual defects can form FESs (defect-free surface states) [31]. On the other hand, by changing the ratio of $\left|\tau_{a}\right| /\left|\tau_{b}\right|$, one can also obtain FESs (Shockley-like surface states).

In order to explore the topological nature of all FESs, we have calculated the quasienergy spectra and the Zak phases. Our results indicate that the Shockley-like surface state is a topological FES and the defect-free surface state is a nontopological FES. However, in our system, although topological and nontopological edge states can exist for the same parameters, they cannot appear in the same spectral gap. Without any embedded or nonlinearity-induced defects, these edge states originate from the interplay between the bulk band topology and periodic modulations. We have derived analytically the boundaries between different topological phases and have verified these results numerically. We believe our work provides perspectives for topological photonics governed by periodic modulations, and can be employed for a control of topological phase transitions. Although our analysis has been performed for arrays of periodically curved optical waveguides, it can be applicable to other lattice systems such as ultracold atoms in optical lattices [42,43], photonic crystals [18], and discrete quantum walks $[44,45]$.

At last, we would like to point out that, if a local defect is introduced, unlike our Floquet edge states, topological and nontopological edge states may appear in the same spectral gap [46].

\section{ACKNOWLEDGMENTS}

This work was supported by the National Natural Science Foundation of China (NNSFC) under Grants No. 11574405 and No. 11465008, and by the Australian Research Council.

Both B.Z. and H.Z. made equal contributions. 
[1] L. Lu, J. D. Joannopoulos, and M. Soljaclc, Topological photonics, Nat. Photon. 8, 821 (2014).

[2] F. D. M. Haldane and S. Raghu, Possible Realization of Directional Optical Waveguides in Photonic Crystals with Broken Time-reversal Symmetry, Phys. Rev. Lett. 100, 013904 (2008).

[3] S. Raghu and F. D. M. Haldane, Analogs of quantum-Hall-effect edge states in photonic crystals, Phys. Rev. A 78, 033834 (2008).

[4] Z. Wang, Y. D. Chong, J. D. Joannopoulos, and M. Soljacic, Observation of unidirectional backscattering-immune topological electromagnetic states, Nature (London) 461, 772 (2009).

[5] R. O. Umucalilar and I. Carusotto, Artificial gauge field for photons in coupled cavity arrays, Phys. Rev. A 84, 043804 (2011).

[6] K. J. Fang, Z. F. Yu, and S. H. Fan, Realizing effective magnetic field for photons by controlling the phase of dynamic modulation, Nat. Photon. 6, 782 (2012).

[7] A. B. Khanikaev, S. H. Mousavi, W. K. Tse, M. Kargarian, A. H. MacDonald, and G. Shvets, Photonic topological insulators, Nat. Mater. 12, 233 (2013).

[8] Y. E. Kraus, Y. Lahini, Z. Ringel, M. Verbin, and O. Zilberberg, Topological States and Adiabatic Pumping in Quasicrystals, Phys. Rev. Lett. 109, 106402 (2012).

[9] M. C. Rechtsman, J. M. Zeuner, Y. Plotnik, Y. Lumer, D. Podolsky, F. Dreisow, S. Nolte, M. Segev, and A. Szameit, Photonic Floquet topological insulators, Nature (London) 496, 196 (2013).

[10] M. Hafezi, S. Mittal, J. Fan, A. Migdall, and J. M. Taylor, Imaging topological edge states in silicon photonics, Nat. Photon. 7, 1001 (2013).

[11] G. Q. Liang and Y. D. Chong, Optical Resonator Analog of a Two-dimensional Topological Insulator, Phys. Rev. Lett. 110, 203904 (2013).

[12] M. Pasek and Y. D. Chong, Network models of photonic Floquet topological insulators, Phys. Rev. B 89, 075113 (2014).

[13] W. C. Hu, J. C. Pillay, K. Wu, M. Pasek, P. P. Shum, and Y. D. Chong, Measurement of a Topological Edge Invariant in a Microwave Network, Phys. Rev. X 5, 011012 (2015).

[14] L. H. Wu and X. Hu, Scheme for Achieving a Topological Photonic Crystal by Using Dielectric Material, Phys. Rev. Lett. 114, 223901 (2015).

[15] C. He, X. C. Sun, X. P. Liu, M. H. Lu, Y. Chen, L. Feng, and Y. F. Chen, Photonic topological insulator with broken time-reversal symmetry, Proc. Natl. Acad. Sci. U.S.A. 113, 4924 (2016).

[16] F. Gao, Z. Gao, X. H. Shi, Z. J. Yang, X. Lin, H. Y. Xu, J. D. Joannopoulos, M. Soljacic, H. S. Chen, L. Lu, Y. D. Chong, and B. L. Zhang, Probing topological protection using a designer surface plasmon structure, Nat. Commun. 7, 11619 (2016).

[17] Y. Lumer, Y. Plotnik, M. C. Rechtsman, and M. Segev, Selflocalized States in Photonic Topological Insulators, Phys. Rev. Lett. 111, 243905 (2013).

[18] J. M. Zeuner, M. C. Rechtsman, Y. Plotnik, Y. Lumer, S. Nolte, M. S. Rudner, M. Segev, and A. Szameit, Observation of a Topological Transition in the Bulk of a Non-Hermitian System, Phys. Rev. Lett. 115, 040402 (2015).

[19] M. S. Rudner, N. H. Lindner, E. Berg, and M. Levin, Anomalous Edge States and the Bulk-edge Correspondence for Periodically Driven Two-dimensional Systems, Phys. Rev. X 3, 031005 (2013).
[20] A. Gomez-Leon and G. Platero, Floquet-Bloch Theory and Topology in Periodically Driven Lattices, Phys. Rev. Lett. 110, 200403 (2013).

[21] A. P. Slobozhanyuk, A. N. Poddubny, A. E. Miroshnichenko, P. A. Belov, and Yu. S. Kivshar, Subwavelength Topological Edge States in Optically Resonant Dielectric Structures, Phys. Rev. Lett. 114, 123901 (2015).

[22] C. Poli, M. Bellec, U. Kuhl, F. Mortessagne, and H. Schomerus, Selective enhancement of topologically induced interface states in a dielectric resonator chain, Nat. Commun. 6, 6710 (2015).

[23] D. Leykam, M. C. Rechtsman, and Y. D. Chong, Anomalous Topological Phases and Unpaired Dirac Cones in Photonic Floquet Topological Insulators, Phys. Rev. Lett. 117, 013902 (2016).

[24] Y. G. Ke, X. Z. Qin, F. Mei, H. H. Zhong, Yu. S. Kivshar, and C. Lee, Topological phase transitions and Thouless pumping of light in photonic waveguide arrays, Laser Photon. Rev. 10, 995 (2016).

[25] L. J. Maczewsky, J. M. Zeuner, S. Nolte, and A. Szameit, Observation of photonic anomalous Floquet topological insulators, Nat. Commun. 8, 13756 (2017).

[26] S. Mukherjee, A. Spracklen, M. Valiente, E. Andersson, P. Ohberg, N. Goldman, and R. R. Thomson, Experimental observation of anomalous topological edge modes in a slowly driven photonic lattice, Nat. Commun. 8, 13918 (2017).

[27] Y. Hatsugai, Chern Number and Edge States in the Integer Quantum Hall Effect, Phys. Rev. Lett. 71, 3697 (1993).

[28] Y. Hatsugai, Edge states in the integer quantum Hall effect and the Riemann surface of the Bloch function, Phys. Rev. B 48, 11851 (1993).

[29] M. Hafezi, Measuring Topological Invariants in Photonic Systems, Phys. Rev. Lett. 112, 210405 (2014).

[30] S. Mittal, S. Ganeshan, J. Y. Fan, A. Vaezi, and M. Hafezi, Measurement of topological invariants in a 2D photonic system, Nat. Photon. 10, 180 (2016).

[31] I. L. Garanovich, A. A. Sukhorukov, and Yu. S. Kivshar, Defectfree Surface States in Modulated Photonic Lattices, Phys. Rev. Lett. 100, 203904 (2008).

[32] A. Szameit, I. L. Garanovich, M. Heinrich, A. A. Sukhorukov, F. Dreisow, T. Pertsch, S. Nolte, A. Tunnermann, and Yu. S. Kivshar, Observation of Defect-free Surface Modes in Optical Waveguide Arrays, Phys. Rev. Lett. 101, 203902 (2008).

[33] W. P. Su, J. R. Schrieffer, and A. J. Heeger, Solitons in Polyacetylene, Phys. Rev. Lett. 42, 1698 (1979).

[34] J. K. Asbóth, L. Oroszlány, and A. Pályi, A Short Course on Topological Insulators, Lecture Notes in Physics Vol. 919 (Springer, Berlin, 2016).

[35] Yu. S. Kivshar and S. K. Turitsyn, Spatiotemporal pulse collapse on periodic potentials, Phys. Rev. E 49, R2536 (1994).

[36] S. Ryu, A. P. Schnyder, A. Furusaki, and A. W. W. Ludwig, Topological insulators and superconductors: Tenfold way and dimensional hierarchy, New J. Phys. 12, 065010 (2010).

[37] S. Ganeshan, K. Sun, and S. Das Sarma, Topological Zeroenergy Modes in Gapless Commensurate Aubry-André-Harper Models, Phys. Rev. Lett. 110, 180403 (2013).

[38] N. Malkova, I. Hromada, X. S. Wang, G. Bryant, and Z. G. Chen, Transition between Tamm-like and Shockley-like surface states in optically induced photonic superlattices, Phys. Rev. A 80, 043806 (2009). 
[39] N. Malkova and C. Z. Ning, Interplay between Tamm-like and Shockley-like surface states in photonic crystals, Phys. Rev. B 76, 045305 (2007).

[40] N. Malkova, I. Hromada, X. S. Wang, G. Bryant, and Z. G. Chen, Observation of optical Shockley-like surface states in photonic superlattices, Opt. Lett. 34, 1633 (2009).

[41] J. Zak, Berry's Phase for Energy-Bands in Solids, Phys. Rev. Lett. 62, 2747 (1989).

[42] M. Atala, M. Aidelsburger, J. T. Barreiro, D. Abanin, T. Kitagawa, E. Demler, and I. Bloch, Direct measurement of the Zak phase in topological Bloch bands, Nat. Phys. 9, 795 (2013).

[43] E. J. Meier, F. A. An, and B. Gadway, Observation of the topological soliton state in the Su-Schrieffer-Heeger model, Nat. Commun. 7, 13986 (2016).
[44] F. Cardano, A. D’Errico, A. Dauphin, M. Maffei, B. Piccirillo, C. de Lisio, G. De Filippis, V. Cataudella, E. Santamato, L. Marrucci, M. Lewenstein, and P. Massignan, Detection of Zak phases and topological invariants in a chiral quantum walk of twisted photons, Nat. Commun. 8, 15516 (2017).

[45] E. Flurin, V. V. Ramasesh, S. Hacohen-Gourgy, L. S. Martin, N. Y. Yao, and I. Siddiqi, Observing Topological Invariants Using Quantum Walks in Superconducting Circuits, Phys. Rev. X 7, 031023 (2017).

[46] C. He, M. H. Lu, W. W. Wan, X. F. Li, and Y. F. Chen, Influence of boundary conditions on the one-way edge modes in two-dimensional magneto-optical photonic crystals, Solid State Commun. 150, 1976 (2010). 